\title{
Circadian Control of Antibacterial Immunity: Findings from Animal Models
}

\author{
Landry L. Tsoumtsa, Cedric Torre and Eric Ghigo * \\ Centre National de la Recherche Scientifique UMR 7278, IRD198, Institut National de la Santé et de la Recherche Médicale \\ U1095, Institut Hospitalier Universitaire Méditerranée-Infection, Aix-Marseille Université, Marseille, France
}

Most of the biological functions, including the immune system, are linked to circadian rhythms in living organisms. Changes occurring to biological parameters as the result of these circadian rhythms can therefore affect the outcome of a disease. For decades, model organisms have proven to be a great tool to understanding biological mechanisms such as circadian cycle and immunity. In this review, we created an inventory of the use of model organisms in order to decipher the relation between circadian rhythms and antibacterial immunity.

Keywords: antibacterial, bacteria, circadian, drosophila, mice, chrono-immunology

\section{INTRODUCTION}

OPEN ACCESS

Edited by:

Brice Rotureau,

Institut Pasteur, France

Reviewed by:

Geanncarlo Lugo-Villarino, Institut de Pharmacologie et de Biologie Structurale/Centre National de la Recherche Scientifique, France

Richard J. Q. McNally,

Newcastle University, UK

Massimo Mallardo,

University of Naples Federico II, Italy

*Correspondence:

Eric Ghigo

eric.ghigo@univ-amu.fr

Received: 07 March 2016 Accepted: 27 April 2016

Published: 10 May 2016

Citation:

Tsoumtsa LL, Torre $C$ and Ghigo E

(2016) Circadian Control of

Antibacterial Immunity: Findings from

Animal Models.

Front. Cell. Infect. Microbiol. 6:54.

doi: 10.3389/fcimb.2016.00054
The use of animals as models is justified for several reasons, including physiological similarity and scientific evidence of biological mechanism conservation between species. Animals share the same environmental conditions as humans and are exposed to environmental pathogens which are similar to those responsible for human diseases. Animals often serve, therefore, as models in immunology in order to investigate pathogenesis relating to bacterial infection. In addition, they are also used to explore host defense mechanisms in response to infection (Conti et al., 2014). Animals are also subject to transitions between day and night, and their immune systems are shown to adapt to these transitions. For example, $8 \%$ of macrophage transcripts are produced in a circadian manner (Keller et al., 2009), Th17 cell differentiation in mice is under circadian control via nuclear receptor ROR $\gamma \tau$ transcription (Yu et al., 2013), and the secretion of TNF $\alpha$ and IL-6 peak in mouse macrophages during the resting phase (Keller et al., 2009). This rhythmic expression of immune components reflects the daily differences between the immune systems of different living organisms.

In this mini-review we aim to review the findings from animal models, with a particular focus on mice and drosophila, which provide links between the antibacterial immune response and circadian rhythms. In addition, we also discuss the contribution of emerging animal models in the study of the relationship between the circadian cycle and anti-bacterial response.

\section{MOLECULAR BASIS OF THE CIRCADIAN RHYTHM}

Daily oscillations of physiological parameters such as blood pressure, body temperature and hormone secretion levels highlight the existence of circadian rhythms (Lamont et al., 2007; Zhang et al., 2014). These rhythms are found in most living organisms, from cyanobacteria to humans. They allow organisms to anticipate and adapt to nycthemeral variations imposed by the rotation of the earth (Merbitz-Zahradnik and Wolf, 2015). Circadian rhythm periods are 
approximately $24 \mathrm{~h}$ long. They are generated by internal biological oscillators. The main oscillator, termed the central oscillator, is the suprachiasmatic nucleus (SCN), which is located at the base of the hypothalamus. Peripheral oscillators are present in many cells, tissues and organs (Dardente and Cermakian, 2005). The role of the SCN is to coordinate and synchronize different oscillators (Ko and Takahashi, 2006). Genetic screening and the study of mutants have identified a set of genes called the "clock genes," which are the molecular basis of rhythm generation. Clock genes interact by forming post-transcriptional and post-translational regulatory loops, creating a 24-h oscillation. In the main loop, the Clock/Bmal1 heterodimer, which constitutes positive limb, promotes Per and Cry transcription during the daytime. PER and CRY proteins are synthesized in the cytoplasm, dimerize and are translocated into the nucleus where they accumulate. In time, this accumulation will repress their own transcription by negative feedback on the Clock/Bmal-1 complex (Ko and Takahashi, 2006). Post-translational modifications, such as phosphorylation or degradation of PER by CSKNI $\varepsilon / \Delta$, can also regulate the accumulation of the PER/CRY complex in the nucleus (Curtis et al., 2014). In some organisms, such as Drosophila, TIM binds to the PER/CRY complex and is translocated into the nucleus (Hastings and Herzog, 2004). Other regulatory loops have also been described, including one that involves the nuclear receptors Rev-erb $\alpha$ and Ror $\alpha$, which compete to bind to ROREs (Ror Response Elements). Rev-erb $\alpha$ binding downregulates Bmal-1 expression while Ror $\alpha$ binding upregulates Bmal-1 expression (Scheiermann et al., 2013). Although the clock genes can vary between species, the molecular organization, structure and function of these genes are highly conserved (Paranjpe and Sharma, 2005).

In mice and Drosophila, the two most commonly used model organisms, the molecular architecture of these feedback loops shows the presence of a $\beta$-Helix Loop Helix domain on Bmal1 and Clock transcripts. This enables binding on D and E-box motifs of both the clock genes and a set of genes involved in other biological processes. Therefore, binding to these motifs induces their rhythmic expression and directly drives these biological processes. These genes are known as "Clock Control Genes" (CCG) (Logan and Sarkar, 2012; Wang et al., 2013). Among the identified CCG are several immune-related genes including Stat3, Stat-5, Erg-1, Nf-kB, and Tlr-9 (Logan and Sarkar, 2012; Silver et al., 2012). Therefore, fluctuations in clocks genes expression lead to variation in the expression of these immune-related genes, which in turn may impact the capacity of the immune system. However, mediation by transcriptional promoters is not the only form of communication between circadian rhythms and the immune response. In murine models, it has been demonstrated that CLOCK forms a complex with the p65 subunit of NF-кB. Clock overexpression increases phosphorylation, and acetylation of the p65 subunit leads to an enhanced transcriptional activity of $N f-k b$ (Spengler et al., 2012). Furthermore, circadian secretion

Abbreviations: Zeitgeber Time (ZT), Corresponding to an experimental time referring to the onset of a zeitgeber (an environmental cue) such as Light-Dark cycle that synchronizes the internal clocks; Circadian Time (CT), Corresponding to an experimental time without any zeitgeber. of melatonin, a clock-controlled humoral factor, may function to modulate the expression of immune-related genes (Deng et al., 2006).

\section{EVIDENCE OF DAILY VARIATION IN THE IMMUNE RESPONSE DURING BACTERIAL INFECTION}

Circadian variation in the ability to combat bacteria was highlighted many years ago. In 1969, researchers proved that, depending on when the infection occurs, circadian fluctuations in the mammalian immune system over the course of the day can lead to changes in the host's response. In this study, mice were subcutaneously inoculated with Diplococcus pneumoniae at different times: 4 a.m., 8 a.m., 12 noon and 8 p.m. (real time). Regardless of the dose of bacteria administered, the mice infected at 4 a.m. showed higher survival rates than the mice infected at 8 a.m., 12 noon and 8 p.m. (Feigin et al., 1969). A few years later, Wongwiwat and colleagues inoculated mice by intraperitoneally with two strains of pneumococcus (type I, A5 strain and non-encapsulated R36NC strains) and observed that, for mice infected at 4 a.m., it took longer to reach an intense level of bacteremia $\left(10^{8}\right.$ bacteria $\left./ \mathrm{ml}\right)$ than the time needed for mice infected at 4 p.m. This led to a higher mortality rate in the mice infected at 4 p.m. compared to those infected at 4 a.m. (Wongwiwat et al., 1972). Both studies clearly showed that in mice, antibacterial response levels can differ according to the time of the challenge. This is likely to be due to the presence of a rhythmic phenomenon in one or more biological process involved in the antibacterial response. Possible mechanisms include the daily difference in hormone and/or enzyme secretion by host during infection. Although Wongwiwat and colleagues failed to identify the specific mechanisms underlying the periodicity of the antibacterial response, they nevertheless demonstrated that an intact adrenal gland is needed for a rhythmic resistance to be observed in mouse survival subsequent to pneumococcal infection, since adrenalectomized mice did not display this rhythmic resistance. As adrenal cortex function displays a circadian rhythm, this proves a direct link between circadian rhythm and resistance to bacteria through adrenal cortex hormones. Recently, a histological analysis of the caeca from mice infected by oral gavage with Salmonella Typhimurium demonstrated that mice infected during the resting phase presented more severe signs of inflammation than mice infected during the active phase. Inflammatory signs such as submucosal edema, surface erosion, cryptitis, inflammatory exudates and mononuclear infiltrate observed in the caeca of infected mice were associated with an increased expression of the TNF $\alpha$ (pro-inflammatory cytokine), CXCL-1 (neutrophil chemoattractant chemokine ligand 1), LCN-2 (antimicrobial peptide lipocalin-2) genes, the expression levels of which were also shown to be dependent upon the time of infection. Additionally, in keeping with the inflammatory nature of tissues, the caeca from mice infected at 10 p.m. (night, early active phase) were significantly less colonized by S. Typhimurium at both 72 and $78 \mathrm{~h}$ post-infection than the caeca from mice infected at 10 a.m. (day, early rest phase) (Bellet et al., 2013). Therefore, 
these results confirm the existence in mammals of a correlation between the time of infection and host response.

In addition, in order to thoroughly investigate the circadian variation in the ability to combat bacteria, several studies have been conducted using Drosophila melanogaster as a model. Flies kept in 12:12 light-dark conditions (LD flies) and inoculated with Streptococcus pneumoniae during the day at ZT7 (Zeitgeber Time (ZT); ZT0 corresponding to lights ON and ZT12 corresponding to lights OFF), die faster than flies infected during the night at ZT19 (Stone et al., 2012). Thus, flies are more resistant to bacterial infection when infected overnight. Similarly, $10 \mathrm{~h}$ after inoculation, DD flies (kept in constant darkness) infected at CT5 (Circadian Time 5) exhibited a higher bacterial load with a poor survival rates than flies infected at CT17 (Lee and Edery, 2008). How can this difference been explained? To answer this question, the authors analyzed the post-infection expression of antimicrobial peptide (AMP) genes such as attacin A, defensine, diptericin, drosocin, drosomycin and genes from Pathogen Recognition Receptor (PRR) signaling pathways such as Pgrp-SA, Pgrp-LC, Pgrp-LB, and imd. The results showed that only the Pgrp-sa and $d r c$ expression patterns differed based upon time of infection. The authors therefore concluded that, based on the time of infection, the circadian rhythm selectively regulates the activation of a limited number of innate immune-related genes which are most likely associated with the production of anti-microbial peptides, found to correlate with bacterial growth kinetics and fly survival rates (Lee and Edery, 2008). Overall, these data reflect the ability of the immune systems of living organisms to adapt to circadian cues. There is a temporal resistance against bacteria which can be explained by circadian modulation on the expression of some immune genes. In mice, the immune system appears to work as if immune alertness fades during the resting phase (day-time in mice), when the animals are less likely to encounter pathogens (Scheiermann et al., 2013) but, as shown by Figure 1, this statement doesn't fit with all living beings. Hence, there is a need to choose the correct model for experiments based on expected outcomes.

\section{THE EFFECT OF CLOCK GENES ON MODULATING THE IMMUNE RESPONSE TO BACTERIA}

The development of genetic engineering tools (RNA interference, Knock-Out) has led to the increased use of animal models such as mice and drosophila in chrono-immunology, because

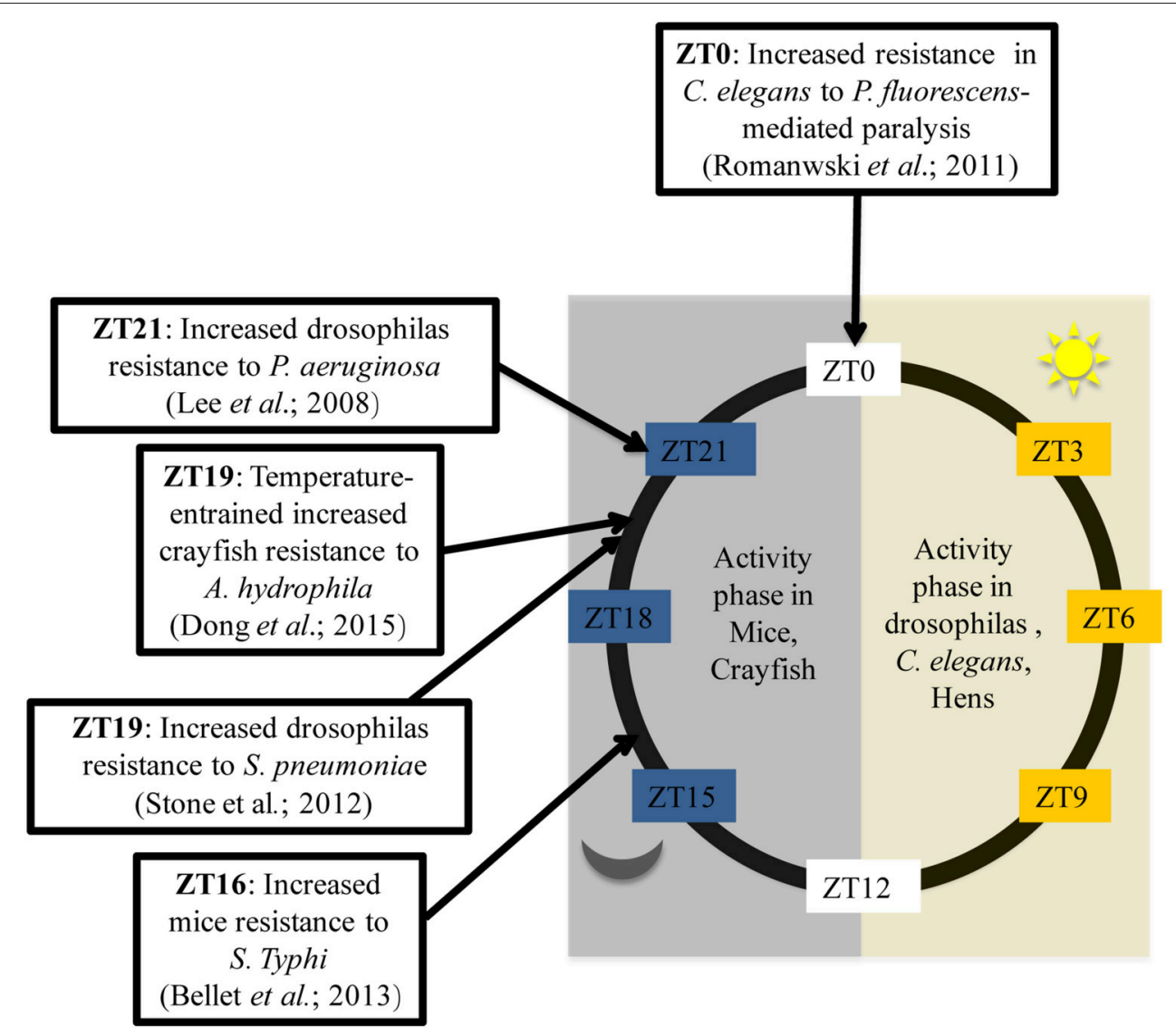

FIGURE 1 | Time of day higher tolerance to bacterial infection in mice, Drosophilas, $\boldsymbol{C}$. elegans, and crayfish. ZT is corresponding to an experimental time referring to the onset of a zeitgeber (light or temperature). ZTO is the transition time from dark to light while ZT12 is the transition time from light to dark. Activity phase is corresponding to the phase where movements or displacements are most recorded. 
TABLE 1 | Clock genes mutations consequences for antibacterial immunity.

\begin{tabular}{|c|c|c|c|c|}
\hline Organisms & Genes knock down & Effects on immunity & Bacterial challenger & References \\
\hline \multirow[t]{4}{*}{ Drosophila } & Clock & Increases resistance & $P$. aeruginosa & Lee and Edery, 2008 \\
\hline & Per-2 & Increases susceptibility & $\begin{array}{l}\text { S.pneumoniae, } P \text {. aeruginosa } \\
\text { L. monocytogenes }\end{array}$ & $\begin{array}{l}\text { Shirasu-Hiza et al., 2007; Lee and Edery, } \\
2008\end{array}$ \\
\hline & Tim & Pathogen-specific & $\begin{array}{l}\text { Increase susceptibility to } \\
\text { S. pneumonia S. marcescens }\end{array}$ & Shirasu-Hiza et al., 2007 \\
\hline & & & Enhanced resistance to $P$. aeruginosa & Lee and Edery, 2008 \\
\hline \multirow[t]{5}{*}{ Mice } & Clock & $\begin{array}{l}\text { Induces less Pro-inflammatory } \\
\text { cytokines production }\end{array}$ & S. typhimurium & Bellet et al., 2013 \\
\hline & Per-2 & $\begin{array}{l}\text { Increases resistance to LPS } \\
\text { endotoxin shock' }\end{array}$ & LPS & Liu et al., 2006 \\
\hline & Bmal-1 & $\begin{array}{l}\text { Increases susceptibility to LPS } \\
\text { endotoxin shock }\end{array}$ & LPS & Curtis et al., 2015 \\
\hline & & Increases susceptibility & L. monocytogenes & Nguyen et al., 2013 \\
\hline & Cry & $\begin{array}{l}\text { Upregulates pro-inflammatory } \\
\text { cytokines genes expression }\end{array}$ & LPS & Narasimamurthy et al., 2012 \\
\hline
\end{tabular}

they provide an opportunity to directly observe the effects of depleting certain circadian factors, such as clock genes, during the bacterial infection (Table 1). In the following reported experiments, majors clock genes were experimentally knockedout in order to mimic desynchronized systems. Both wild type (WT) D. melanogaster infected at CT17 and Clock (-/-) $D$. melanogaster were shown to be resistant to P. aeruginosa infection. However, WT D. melanogaster infected at CT17 were more resistant to $P$. aeruginosa infection than WT $D$. melanogaster infected at CT5 (Lee and Edery, 2008). Likewise, $72 \mathrm{~h}$ after infection with $S$. Typhimurium, Clock (-/-) mice exhibited a bacterial load similar to that found in WT mice infected overnight, but lower than that in WT mice infected during the day (Bellet et al., 2013). Therefore, the absence of Clock alters the previously described daily variation of susceptibility to bacteria depending on the time of infection, and allows the immune system to be on high alert against pathogens throughout the day. Furthermore, during infection, Clock (-/-) mice produce fewer pro-inflammatory cytokines (TNF $\alpha$, IFN $\beta$, IL-6, IL-1 $\beta$ ) than WT mice (Bellet et al., 2013). In contrast, Cry $(-/-)$ mice produce more cytokines (IL-6, TNF $\alpha$ ) than WT mice (Narasimamurthy et al., 2012). Thus, pro-inflammatory cytokines secretion is controlled via the expression of clock genes, suggesting that the circadian rhythm can modulate antibacterial response. In another study using mice as models, regardless of the time at which derived Escherichia coli (E. coli) LPS was injected, deletion of the Clock partner, Bmal-1, decreased the survival rate of mice following LPS endotoxic shock (Curtis et al., 2015). The same results were obtained after intraperitoneal infection of Bmal-1 (-/-) mice with Listeria monocytogenes. Indeed, compared to the control, Bmal-1 (-/-) mice exhibited a decrease on the survival rate during L. monocytogenes infection, explained by the presence in mice of an Bmal-1 dependant rhythmic mobilization of Ly6C ${ }^{\text {hi }}$ monocytes (Nguyen et al., 2013). Per-2 $(-/-)$ mice present significantly increased resistance to E. coli LPS endotoxic shock, as $50 \mathrm{~h}$ after LPS administration, all Per-2 $(-/-)$ mice survived the endotoxic shock, while the WT mice died (Liu et al., 2006). Indeed, Per-2 plays an immune-regulatory role in the production of pro-inflammatory cytokines such as INF $\gamma$ and IL-1 but not in the production of others cytokines (IL6, IL-10, TNF $\alpha$ ) (Arjona and Sarkar, 2006; Liu et al., 2006). The decrease in INF $\gamma$ production may be due to the low number of NK cells or the defective NKT function observed in Per-2 (-/-) mice. Thus, Per-2 appears to offer circadian control in the innate immune response by acting as a regulator of the production and function of NK cells. In D. melanogaster, deletion of Per-2 renders flies more sensitive to $S$. pneumoniae, $P$. aeruginosa and Listeria monocytogenes. Ten hours after infection, the bacterial load determined by CFU (Colony Forming Units) counts was higher in Per-2 (-/-) than in Clock $(-/-)$ mice (Shirasu-Hiza et al., 2007; Lee and Edery, 2008). The aforementioned studies appear to give a contradictory role to Per-2 in the antibacterial immune response in mice and in Drosophila. In mice, however, the challenge was performed using the Gram-negative bacteria ligand LPS, which is certainly immunogenic but is endotoxic and may induce a less complete immune response than the entire bacteria. It would, therefore, be of great interest to conduct further experiments to explore the response of Per-2 (-/-) mice to entire bacteria.

In regards to Tim, Lee and colleagues found a survival advantage in Tim (-/-) flies infected with S. pneumonia, while Shirasu-Hiza described increased susceptibility of Tim (-/-) flies to $P$. aeruginosa infection compared to WT flies (ShirasuHiza et al., 2007; Lee and Edery, 2008). Interestingly, it appears that Tim involvement in the immune response to bacteria could be pathogen-dependent. Indeed, researchers infected Tim (-/-) D. melanogaster by injecting four different strains of bacteria. Two days after infection with $S$. pneumoniae and Serratia marcescens, Tim (-/-) contained more bacteria than WT D. melanogaster, while 2 days after infection with Burkholderia cepacia and S. typhimurium, Tim $(-/-)$ s contained the same levels of bacteria as WT (Stone et al., 2012). The pathogendependent actions of Tim may be linked to specific defense mechanisms which the flies engage in the presence of a specific 
type of bacteria. Recently, Tim has been shown to regulate the phagocytosis of bacteria in Drosophila but TIM is degraded by light. This explains the increased susceptibility to bacteria observed during the day and the significant phagocytic activity against bacteria observed during the night in Drosophila. Indeed, Tim (-/-) D. melanogaster have significantly lower phagocytic activity than WT flies after Staphylococcus aureus infection, but no difference is observed during E. coli infection (Stone et al., 2012). Therefore, Tim regulates phagocytosis in a pathogenspecific manner. In their results, while phagocytosis is shown to be regulated by Tim expression, melanization and antimicrobial peptide gene expression are not shown to be controlled either by Tim expression or by the circadian clock machinery (Stone et al., 2012).

Key clock gene inhibitions always have immediate consequences on the antibacterial response, revealing a circadian control of the host's response to bacterial infection. However, mechanisms and factors allowing for this circadian control are not yet fully understood, particularly because only a few clock genes have already been investigated despite the fact that the circadian machinery in mammals involves more than a dozen genes. Of particular interest is Rev Erb $\alpha$ and Roryt, which have been shown through in vitro studies on mice cells to have a modulatory effect on the inflammatory function of macrophages and a regulatory role on Th17 lymphocyte differentiation, respectively (Yu et al., 2013; Sato et al., 2014). Thus, additional study is required to fully determine the actors, effectors and mechanisms responsible for the connection between the circadian system and the host's response to bacteria.

\section{INFLUENCE OF L/D ENTRAINED RHYTHM ON BACTERIAL CHALLENGE}

Circadian rhythms can be influenced by environmental factors known as zeitgebers. The most important zeitgeber is light. Light cues give the internal clock of the organism information required to reset its phase (El Allali et al., 2013). Light/dark cycling keeps this rhythm synchronized with the environment. The majority of the experiments mentioned above were carried out using animals kept in a 12:12 light/dark cycle. This condition appears to be the most representative of the natural light cycle which living things, including human beings, are subject to in real life conditions. On the other hand, maintaining model organisms under constant conditions (light or dark) may induce desynchronization, with dramatic modifications to the circadian rhythm period, amplitude and/or acrophase. This "free run" of the rhythm can be found in humans with clinical conditions such as cancer, metabolic and autoimmune diseases (Lamont et al., 2007). For example, patients in intensive care units suffer from a disruption to the circadian rhythm often caused by the lack of natural light, noise and medication. This disruption is marked by sleep disturbances and is significantly associated with the occurrence of severe sepsis (Brainard et al., 2015). Furthermore, the immune machinery regulated by circadian rhythms is perfectly synchronized with environmental cues. This regulation may be controlled by melatonin secretion during the dark period. Immune cells contain melatonin receptors and binding melatonin to these receptors promotes the expression of interleukins and IFN $\gamma$ (Berger, 2008). Likewise, the absence of light stimulates the expression of IFN $\gamma$, which is also mediated by melatonin secretion. As demonstrated by Lundkvist in 1999, IFN $\gamma$ secretion is greater in the suprachiasmatic nuclei of DD rats compared to LD rats (Lundkvist et al., 1999). Lundkvist also observed a loss of rhythmic IFN $\gamma$ secretion in the CNS of DD rats. In addition, in the absence of melatonin secretion due to constant light exposure, the phagocytic activity of LL rat neutrophils in response to Escherichia coli remains rhythmic but is significantly lower than in LD rats. In LD rats, the phagocytic activity of neutrophils increased during the dark period (Hriscu et al., 2002-2003). In Drosophila maintained in constant darkness, fly survival rate depends on the time of infection, which varies in a circadian manner. However, regardless of the time of infection, DD Tim, Cyc and Clock (-/-) flies survive significantly less often upon infection with $P$. aeruginosa than LD Tim, Cyc and Clock (-/-) flies. However, DD Per (-/-) flies survive better than LD Per (-/-) flies (Lee and Edery, 2008). In conclusion, synchronized organisms respond more efficiently to bacterial infection than those which are desynchronized.

\section{CONTRIBUTION OF EMERGING ANIMAL MODELS}

Major clock genes are highly conserved in living beings. Indeed, Clock, Per and Tim homologs are present in C. elgans, crayfish and chickens. (Escamilla-Chimal et al., 2010; Temmerman et al., 2011; Zhang et al., 2016). These alternative models thus became increasingly used to study the link between circadian rhythms and antibacterial immunity. In Caenorhabditis elegans, fast paralytic killing caused by Pseudomonas aeruginosa or Pseudomonas fluorescens occurred more quickly when worms were infected during the night (Romanowski et al., 2011). Less conventional models have also been used to analyze this phenomenon. Temperature affects circadian variations in the immune status of crayfish. Indeed, when placed on a temperature cycle (between $18^{\circ} \mathrm{C}$ and $24^{\circ} \mathrm{C}$ ), crayfish infected with Aeromonas hydrophila at CT19 presented a significantly lower bacterial load $12 \mathrm{~h}$ after infection compared with crayfish infected at CT5. This gave the crayfish infected at CT19 a survival advantage (Dong et al., 2015). Furthermore, a microarray analysis of gene expression in the caecum from resistant (3.50 log CFU) or sensitive (1.39 log CFU) chickens to Campylobacter jejuni found that Clock expression was significantly associated with resistance to C. jejuni ( $\mathrm{Li}$ et al., 2010). Taken as a whole, these studies demonstrate that circadian rhythms play an important role in the host's response to bacterial infections Figure 1. The use of models which are resistant to bacterial infection may provide additional information. Recently, it has been shown that the immortal flatworms, planarians, are resistant to a large number of pathogenic microorganisms (Abnave et al., 2014). Indeed, planarians are able to eliminate 18 different strains of pathogenic bacteria (Abnave et al., 2014). Two species of planarians have been studied: Schmidtea mediterranea and Dugesia japonica. 
One mechanism explaining planarians' resistance to bacteria involves Morn-2. This resistance mechanism has been proven to be transferable to humans. Morn-2 forced expression in human macrophages reduced by approximately 70\% Mycobacterium tuberculosis proliferation in macrophages (Abnave et al., 2014). However, the role of or link between circadian cycle and bacterial resistance in planarians has not yet been investigated. Furthermore, planarians kept in 12:12 light/dark conditions exhibit diurnal variation in the secretion levels of melatonin and serotonin (Itoh et al., 1999; Itoh and Igarashi, 2000). There is also substantial evidence of the existence of an internal clock in planarians which controls many physiological processes, such as fission during asexual reproduction (Sheiman et al., 2003). This suggests the possible conservation of several mammalian clock genes in planarians. Therefore, planarians may be a good model for identifying the influence of the circadian clock on resistance factors to bacterial infections.

\section{CONCLUDING REMARKS}

Understanding chrono-immunology may allow the potential benefits to be identified which may improve our ability to efficiently combat bacterial infections. Research using animal models clearly establishes a link between the circadian machinery and antibacterial immunity. Clock genes have proven to be involved in the fight against bacterial invasion, both in vertebrate and invertebrate organisms. Studies conducted thus far are restricted to exploring the impact of the down regulation of such genes on antibacterial response and, yet, it would also be of great interest to examine the over-expression effect on antibacterial response. The use of mutated organisms for circadian clock

\section{REFERENCES}

Abnave, P., Mottola, G., Gimenez, G., Boucherit, N., Trouplin, V., Torre, C., et al. (2014). Screening in planarians identifies MORN2 as a key component in LC3associated phagocytosis and resistance to bacterial infection. Cell Host Microbe 16, 338-350. doi: 10.1016/j.chom.2014.08.002

Arjona, A., and Sarkar, D. K. (2006). The circadian gene mPer2 regulates the daily rhythm of IFN-gamma. J. Interferon Cytokine Res. 26, 645-649. doi: 10.1089/jir.2006.26.645

Bellet, M. M., Deriu, E., Liu, J. Z., Grimaldi, B., Blaschitz, C., Zeller, M., et al. (2013). Circadian clock regulates the host response to Salmonella. Proc. Natl. Acad. Sci. U.S.A. 110, 9897-9902. doi: 10.1073/pnas. 1120636110

Berger, J. (2008). A two-clock model of circadian timing in the immune system of mammals. Pathol. Biol. 56, 286-291. doi: 10.1016/j.patbio.2007.10.001

Brainard, J., Gobel, M., Bartels, K., Scott, B., Koeppen, M., and Eckle, T. (2015). Circadian rhythms in anesthesia and critical care medicine: potential importance of circadian disruptions. Semin. Cardiothorac. Vasc. Anesth. 19, 49-60. doi: 10.1177/1089253214553066

Conti, F., Abnave, P., and Ghigo, E. (2014). Unconventional animal models: a booster for new advances in host-pathogen interactions. Front. Cell. Infect. Microbiol. 4:142. doi: 10.3389/fcimb.2014.00142

Curtis, A. M., Bellet, M. M., Sassone-Corsi, P., and O'neill, L. A. (2014). Circadian clock proteins and immunity. Immunity 40, 178-186. doi: 10.1016/j.immuni.2014.02.002

Curtis, A. M., Fagundes, C. T., Yang, G., Palsson-Mcdermott, E. M., Wochal, P., Mcgettrick, A. F., et al. (2015). Circadian control of innate immunity in genes will allow us to understand the influence of circadian rhythms on human antibacterial defenses and help identify critical circadian factors needed to improve resistance to bacteria. Moreover, constant or alternating conditions between light and darkness, the mode of infection and specific host factors (diurnal or nocturnal activity) had undoubtedly a significant influence upon the results presented above. Thus, it seems important to note that the interaction between the circadian rhythm and antibacterial immunity relies on complex interconnected communication systems depending on environmental factors, the circadian rhythm itself, the host and the bacteria. All these parameters must, therefore, be taken into account when selecting experimental conditions in order to obtain standardized and comparable experiments and, of course, study of this interaction cannot be restricted to the study of clock genes. Overall, animal models should continue to be used as the primary tool for investigating the molecular mechanisms underlying the interaction between circadian rhythms and antibacterial immune responses.

\section{AUTHOR CONTRIBUTIONS}

LT conceptualization, original draft. CT original draft. EG conceptualization, original draft, supervision, resourrces, funding, review and editing.

\section{ACKNOWLEDGMENTS}

The work of our team related to the topic reviewed in this article was supported by CNRS. LT is fellows of "Infectiopole Sud" and the "Agence Universitaire de la Francophonie." CT is fellows of the French Ministry for Research and Technology.

macrophages by miR-155 targeting Bmall. Proc. Natl. Acad. Sci. U.S.A. 112, 7231-7236. doi: 10.1073/pnas.1501327112

Dardente, H., and Cermakian, N. (2005). [How many pieces to build a circadian clock?]. Med. Sci. (Paris). 21, 66-72. doi: 10.1051/medsci/200521166

Deng, W. G., Tang, S. T., Tseng, H. P., and Wu, K. K. (2006). Melatonin suppresses macrophage cyclooxygenase- 2 and inducible nitric oxide synthase expression by inhibiting p52 acetylation and binding. Blood 108, 518-524. doi: 10.1182/blood-2005-09-3691

Dong, C., Bai, S., and Du, L. (2015). Temperature regulates circadian rhythms of immune responses in red swamp crayfish Procambarus clarkii. Fish Shellfish Immunol. 45, 641-647. doi: 10.1016/j.fsi.2015.05.025

El Allali, K., Achaâban, M. R., Bothorel, B., Piro, M., Bouâouda, H., El Allouchi, M., et al. (2013). Entrainment of the circadian clock by daily ambient temperature cycles in the camel (Camelus dromedarius). Am. J. Physiol. Regul. Integr. Comp. Physiol. 304, R1044-R1052. doi: 10.1152/ajpregu.0046 6.2012

Escamilla-Chimal, E. G., Velázquez-Amado, R. M., Fiordelisio, T., and FanjulMoles, M. L. (2010). Putative pacemakers of crayfish show clock proteins interlocked with circadian oscillations. J. Exp. Biol. 213, 3723-3733. doi: $10.1242 /$ jeb. 047548

Feigin, R. D., San Joaquin, V. H., Haymond, M. W., and Wyatt, R. G. (1969). Daily periodicity of susceptibility of mice to pneumococcal infection. Nature 224, 379-380. doi: 10.1038/224379a0

Hastings, M. H., and Herzog, E. D. (2004). Clock genes, oscillators, and cellular networks in the suprachiasmatic nuclei. J. Biol. Rhythms 19, 400-413. doi: $10.1177 / 0748730404268786$ 
Hriscu, M., Saulea, G., Ostriceanu, S., and Baciu, I. (2002-2003). Circadian phagocytic activity in rats under light-dark and constant light regimens. Rom. J. Physiol. 39-40, 17-26.

Itoh, M. T., and Igarashi, J. (2000). Circadian rhythm of serotonin levels in planarians. Neuroreport 11, 473-476. doi: 10.1097/00001756-200002280-00009

Itoh, M. T., Shinozawa, T., and Sumi, Y. (1999). Circadian rhythms of melatoninsynthesizing enzyme activities and melatonin levels in planarians. Brain Res. 830, 165-173. doi: 10.1016/S0006-8993(99)01418-3

Keller, M., Mazuch, J., Abraham, U., Eom, G. D., Herzog, E. D., Volk, H. D., et al. (2009). A circadian clock in macrophages controls inflammatory immune responses. Proc. Natl. Acad. Sci. U.S.A. 106, 21407-21412. doi: 10.1073/pnas.0906361106

Ko, C. H., and Takahashi, J. S. (2006). Molecular components of the mammalian circadian clock. Hum. Mol. Genet. 15 Spec No 2, R271-R277. doi: $10.1093 / \mathrm{hmg} / \mathrm{ddl} 207$

Lamont, E. W., James, F. O., Boivin, D. B., and Cermakian, N. (2007). From circadian clock gene expression to pathologies. Sleep Med. 8, 547-556. doi: 10.1016/j.sleep.2006.11.002

Lee, J. E., and Edery, I. (2008). Circadian regulation in the ability of Drosophila to combat pathogenic infections. Curr. Biol. 18, 195-199. doi: 10.1016/j.cub.2007.12.054

Li, X., Swaggerty, C. L., Kogut, M. H., Chiang, H. I., Wang, Y., Genovese, K. J., et al. (2010). Gene expression profiling of the local cecal response of genetic chicken lines that differ in their susceptibility to Campylobacter jejuni colonization. PLOS ONE 5:e11827. doi: 10.1371/journal.pone.00 11827

Liu, J., Malkani, G., Mankani, G., Shi, X., Meyer, M., Cunningham-Runddles, S., et al. (2006). The circadian clock Period 2 gene regulates gamma interferon production of $\mathrm{NK}$ cells in host response to lipopolysaccharide-induced endotoxic shock. Infect. Immun. 74, 4750-4756. doi: 10.1128/IAI.00287-06

Logan, R. W., and Sarkar, D. K. (2012). Circadian nature of immune function. Mol. Cell. Endocrinol. 349, 82-90. doi: 10.1016/j.mce.2011.06.039

Lundkvist, G. B., Andersson, A., Robertson, B., Rottenberg, M. E., and Kristensson, K. (1999). Light-dependent regulation and postnatal development of the interferon-gamma receptor in the rat suprachiasmatic nuclei. Brain Res. 849, 231-234. doi: 10.1016/S0006-8993(99)02086-7

Merbitz-Zahradnik, T., and Wolf, E. (2015). How is the inner circadian clock controlled by interactive clock proteins?: Structural analysis of clock proteins elucidates their physiological role. FEBS Lett. 589, 1516-1529. doi: 10.1016/j.febslet.2015.05.024

Narasimamurthy, R., Hatori, M., Nayak, S. K., Liu, F., Panda, S., and Verma, I. M. (2012). Circadian clock protein cryptochrome regulates the expression of proinflammatory cytokines. Proc. Natl. Acad. Sci. U.S.A. 109, 12662-12667. doi: 10.1073/pnas.1209965109

Nguyen, K. D., Fentress, S. J., Qiu, Y., Yun, K., Cox, J. S., and Chawla, A. (2013). Circadian gene Bmall regulates diurnal oscillations of Ly6C(hi) inflammatory monocytes. Science 341, 1483-1488. doi: 10.1126/science.12 40636

Paranjpe, D. A., and Sharma, V. K. (2005). Evolution of temporal order in living organisms. J. Circadian Rhythms 3, 7. doi: 10.1186/1740-3391-3-7

Romanowski, A., Migliori, M. L., Valverde, C., and Golombek, D. A. (2011). Circadian variation in Pseudomonas fluorescens (CHA0)-mediated paralysis of Caenorhabditis elegans. Microb. Pathog. 50, 23-30. doi: 10.1016/j.micpath.2010.09.001
Sato, S., Sakurai, T., Ogasawara, J., Takahashi, M., Izawa, T., Imaizumi, K., et al (2014). A circadian clock gene, Rev-erb $\alpha$, modulates the inflammatory function of macrophages through the negative regulation of $\mathrm{Ccl} 2$ expression. J. Immunol. 192, 407-417. doi: 10.4049/jimmunol.1301982

Scheiermann, C., Kunisaki, Y., and Frenette, P. S. (2013). Circadian control of the immune system. Nat. Rev. Immunol. 13, 190-198. doi: 10.1038/nri3386

Sheǐman, I. M., Sakharova, N. I. U., Tiras, K. P., Shkutin, M. F., and Isaeva, V. V. (2003). [Regulation of asexual reproduction in the planarian Dugesia tigrina]. Ontogenez 34, 43-49.

Shirasu-Hiza, M. M., Dionne, M. S., Pham, L. N., Ayres, J. S., and Schneider, D. S. (2007). Interactions between circadian rhythm and immunity in Drosophila melanogaster. Curr. Biol. 17, R353-R355. doi: 10.1016/j.cub.2007.03.049

Silver, A. C., Arjona, A., Walker, W. E., and Fikrig, E. (2012). The circadian clock controls toll-like receptor 9-mediated innate and adaptive immunity. Immunity 36, 251-261. doi: 10.1016/j.immuni.2011.12.017

Spengler, M. L., Kuropatwinski, K. K., Comas, M., Gasparian, A. V., Fedtsova, N., Gleiberman, A. S., et al. (2012). Core circadian protein CLOCK is a positive regulator of NF-кB-mediated transcription. Proc. Natl. Acad. Sci. U.S.A. 109, E2457-E2465. doi: 10.1073/pnas.1206274109

Stone, E. F., Fulton, B. O., Ayres, J. S., Pham, L. N., Ziauddin, J., and Shirasu-Hiza, M. M. (2012). The circadian clock protein timeless regulates phagocytosis of bacteria in Drosophila. PLoS Pathog. 8:e1002445. doi: 10.1371/journal.ppat.1002445

Temmerman, L., Meelkop, E., Janssen, T., Bogaerts, A., Lindemans, M., Husson, S. J., et al. (2011). C. elegans homologs of insect clock proteins: a tale of many stories. Ann. N.Y. Acad. Sci. 1220, 137-148. doi: 10.1111/j.17496632.2010.05927.x

Wang, Z., Wu, Y., Li, L., and Su, X. D. (2013). Intermolecular recognition revealed by the complex structure of human CLOCK-BMAL1 basic helix-loop-helix domains with E-box DNA. Cell Res. 23, 213-224. doi: 10.1038/cr.2012.170

Wongwiwat, M., Sukapanit, S., Triyanond, C., and Sawyer, W. D. (1972). Circadian rhythm of the resistance of mice to acute pneumococcal infection. Infect. Immun. 5, 442-448.

Yu, X., Rollins, D., Ruhn, K. A., Stubblefield, J. J., Green, C. B., Kashiwada, M., et al. (2013). TH17 cell differentiation is regulated by the circadian clock. Science 342, 727-730. doi: 10.1126/science. 1243884

Zhang, R., Lahens, N. F., Ballance, H. I., Hughes, M. E., and Hogenesch, J. B. (2014). A circadian gene expression atlas in mammals: implications for biology and medicine. Proc. Natl. Acad. Sci. U.S.A. 111, 16219-16224. doi: 10.1073/pnas.1408886111

Zhang, Z. C., Wang, Y. G., Li, L., Yin, H. D., Li, D. Y., Wang, Y., et al. (2016). Circadian clock genes are rhythmically expressed in specific segments of the hen oviduct. Poult. Sci. doi: 10.3382/ps/pew051. [Epub ahead of print].

Conflict of Interest Statement: The authors declare that the research was conducted in the absence of any commercial or financial relationships that could be construed as a potential conflict of interest.

Copyright (c) 2016 Tsoumtsa, Torre and Ghigo. This is an open-access article distributed under the terms of the Creative Commons Attribution License (CC BY). The use, distribution or reproduction in other forums is permitted, provided the original author(s) or licensor are credited and that the original publication in this journal is cited, in accordance with accepted academic practice. No use, distribution or reproduction is permitted which does not comply with these terms. 